N. Magnenat Thalmann, H. Kim, A. Egges, S. Garchery. Believability and Interaction in Virtual Worlds. International Multi-Media Modelling Conference, IEEE Computer Society Press, pp. 2-9. January 2005. 


\title{
Believability and Interaction in Virtual Worlds
}

\author{
Nadia Magnenat-Thalmann, HyungSeok Kim, Arjan Egges and Stephane Garchery \\ MIRALab - University of Geneva \\ 24, Rue General-Dufour \\ CH-1211 Geneva, Switzerland \\ \{thalmann,kim,egges,garchery\}@ miralab.unige.ch
}

\begin{abstract}
In this paper we present a discussion about believability for Virtual Environments, emotional simulation and also Embodied Conversational Agents (ECAs). We will discuss about the definition of believability and the three elements of believability environments (immersion, presentation and interaction). We also present a discussion about believability and interfaces. Finally, ECA, emotional and personnality simulation are explained and presented.
\end{abstract}

\section{Introduction}

The goal of the virtual reality system can be stated that it is to simulate the sensory information such that the participant feels that the generated experience is from real-world. The believability of the virtual environment is a term for possible measurement of achieving this goal. In this paper, we will discuss believability issues in Virtual Environments.

The definition of believability is still an open issue. Zeltzer states that Autonomy, Interactivity, and Presence are important elements for the Virtual Environment [52]. These elements are one of the most essential ones to make the virtual world 'realistic' but in terms of believability, a traditional definition of these terms is not sufficient. For the character representation, the believability is often discussed in context of generating behaviors [33]. Believable behavior covers not only realism but also emotions, personality, and intent [12]. We call these additional issues the perceptual believability in comparison to the sensory believability which represents realism in sensory channel. To incorporate and measure these issues, we identify three elements of believable virtual environment as immersion, presentation, and interaction.

\section{- Immersion}

The user can believe that the experience in the virtual world is a real experience if he or she is totally immersed in the virtual environment. Immersion to the virtual environment can be categorized in terms of sensory immersion and perceptual immersion. Sensory immersion is provided by utilizing immersive devices including HMD or CAVE-like systems. Modelling and measuring this kind of immersion has been conducted by utilizing both cognitive surveys and performance indicators $[38,41]$. In other points of view, users are immersed into the virtual world if its semantics are realistic. Sheridan called this element as 'the active imagination in suppressing disbelief (and thus enhanced believability)' [47]. The semantics of a virtual environment consists of emotional elements, personalized elements and goal-oriented elements. If a set of objects and stories have these elements, participants believe the represented world. This level of immersion is often called presence.

\section{- Presentation}

The believability of the virtual environment can be increased if the virtual world is presented as real as real world. Still it is true that non-realistic experience can give enough immersion and enough believability but if it is combined with realistic presentation, it will increase the believability. The realism in the presentation can be discussed in terms of sensory signal level realism and perceptual level realism.

\section{- Interaction}

One of the most important issues in the virtual environment is its interactivity. A realistic interactive system will result in higher believability in normal cases. The realism of the interactivity can be determined by observing its reactive behavior. Realistic reactive behavior in interactivity is related but different from behaviors to induce perceptual immersion. The interactivity is increased if the behavior responds to actions of users in a life-like way. Early psychology research states that virtual charactor should react based on their perceived or assumed goals, beliefs and attitudes [37]. Immersion largely depends on how well this is im- 
plemented, for example through goal-oriented artificial intelligence or emotional behavior simulation [35, 33]. We believe that the perceptual immersion is invoked by goal-oriented intervention of intents, emotions, and personality. The realism of the interaction is defined by the involvement of the user in the virtual environment. For example, factors of presence as defined by Stevens et al. [49] can be re-categorized so that: 1) personal presence, intended aspect of social presence and task factors are components of immersion, 2) unintended aspect of social presence and environmental presence are components of realism in interactivity.

These effects of these elements are not independent. They influence each other in a complex way. In some cases a high level of realism for one area will elaborate the level of believability but if it is combined with a low level of realism on other area, it will decrease the level of believability. Even if the sensory channel has enough realism, it is not sufficient to make the VE believable if the VE does not have believable contents. From another point of view, a VE presented in written text (for example a novel or a book) depending on the quality of the stories.

In the section 2, issues in sensory channel is discussed. Perceptual believability is discussed in the section 3 focused on the emotional issues.

\section{Believability and Interfaces}

In terms of interface, believability can be discussed for each sensory channels. If each sensory channel can reproduce information with enough believable way, the whole virtual environment can be presented in believable way to the participant.

Among primary human sensory channels visual, auditory and haptic has been major elements in terms of interface. In this section, the believability issues of these three major channel are discussed.

\subsection{Realistic visual sensory feedback}

The visual sensory channel is a one of the most important channel to make virtual world believable. For example from the early version of movies, it has given successful believable experiences to audiences using mostly visual information only. Visual channel is the most investigated sensory channel in the virtual reality scene. Issues including modelling and re-producing visual information are investigated since the beginning of the computer graphics in 60's. They are started from the modelling and re-producing the virtual world itself and it is evolved to integrating real and virtual world altogether.

In the virtual environment, an immersion is a technical term describing the extent to which the sensory apparatus of the participant is exposed to the synthetic cues generated by the virtual environment. Depending on the types and number of the devices used one can arbitrary identify different types and levels of immersion.

Visual immersion is achieved through the use of shutter glasses, HMD or CAVE-like system. Various levels of the visual immersion is also achieved by adopting software technologies to simulate the visual cues: stereo- or monoscopic, number of colors, resolution, field of view, focal distance, etc. From the very early beginning of the virtual reality technologies, various immersive visual displays are developed. There have been many work to measure 'sense of presence' for difference visual immersion levels. These are measured in terms of distance/depth perception task performance and easy of use.

To achieve realism in the presentation, most of work has been done to generate images to have image level realism. The image level realism is defined as a state of realism in image with comparison in the real-image in terms of pixel-wise comparison. Realistic shape modelling and realistic illumination modelling fall into this category. Realistic shape modelling is investigated in various levels including capturing real shape using camera, special sensors including laser scanning and representing special features of the shape such as smoothness of the surface. To achieve more realism various representation is investigated from parametric surface model, polygon surface model, point-based model, image-based model, and volumetric model. The realism in the presentation is primarily depends on the amount of data such as number of polygons or resolution of images. There have been many attempts to control the amount of data for real-time realistic visualization. There methods try to minimize the degradation of visual realism whilst reducing amount of data to be processed. There are also a set of work to model realistic illumination models. Starting from the simple point-light model, more complex light environment is investigated including modelling area lights, capturing light environments, simulating complex materials. The biggest issues of visual realism in the presentation is again the measurement. Mainly, the shape related geometric measurements, such as length, area, volume and curvature, are used to measure visual realism. Recently, some work has been done to consider human sensory limitation or perceptual issues such as give more detailed model where human visual sensor can perceive its delicate details.

\subsection{Reproducing auditory information for immer- sive virtual environment}

The audio is as or even more important than the video. If the surround sound is believable to your ear and brain, you will be there but not here, you will be transported. The surrounding sound defines the environment all around you. 
Nevertheless, the problem encountered to give this sense of realism and believability comes from speakers which produce noises and distortions. Plus, higher the number of sources and the complexity of the virtual environment is, higher the rendering of an accurate sound in real-time is complex.

3D spatial audio in virtual environments is a relatively new and wide research topic, although spatial audio in general has been under investigation since the beginning of the last century. Rendering audible space with preserved threedimensional sound illusion is called auralization according to Kleiner [25]. Virtual acoustics include virtual reality aspects such as dynamic listener, dynamic source and acoustic environment specificities as described by Takala $[50,51]$ and Savioja [43], who described general methodology for producing sound effects for animation. For basic knowledge of simulation and rendering of sound in virtual environments, we can refer to Funkhouser [19], Savioja [42], Huopaniemi [23], and the book written by Begault [4]. Some fundamental elements already existent are necessary for a complete spatial audio system including transmission, reflections, reverberation, diffraction, refraction and head related transfer. As can be observed, some of these elements are affected by the position of the sound source relative to the listener (or receiver) and others are affected by the environments itself. Several propagation methods are proposed to simulated sound effect from the sound source to the listener $[1,5,26,29,20,21]$. Most of sound rendering techniques reproduce the sound field for a specific listening point. Binaural and transaural techniques directly attempt to model the sound field at both ears, while techniques based on loudspeaker arrays reconstruct the sound field at the center of the reproduction setup (and usually degrade quickly as the listener moves off-center). Multichannel panning techniques are simple and efficient, but are more limited in imaging quality than Ambisonic techniques. Wave-field synthesis(WFS) is uniquely able to reconstruct the correct wavefronts everywhere inside the listening region and are thus a true multi-user reproduction system. However the inherent complexity of a WFS setup has, to date, prevented its use in virtual environment systems.

The presentation realism of auditory information can be represented based on subjective observations (exchange of opinions between acousticians and musicians in the case of concert halls); energy decay, clarity and binaural aspects.

\subsection{Review of haptic devices in terms of believ- ability}

Until now, haptic sensory feedback is simulated in limited way. Although there have been discussion and illustration on full body haptic reproduction, for example data suite the current technological level is still far away from that goal. Currently, most effort is devoted to simulate realistic presentation of haptic. Among the wide range of systems available, one can define several classes of devices:

\section{- Arm-like devices}

The user grasp a robotic arm with several degrees of freedom that can apply forces. The example of this kind devices are PHANTOM, Dextrous Arm and Freedom 6S [ref]. For this kind of devices, several factors will determine the realism of the simulations. First of all, the scenario of the simulation must include a pen like tool for interacting with the virtual world, as the device cannot reproduce any other kind of tool. The haptic device has to be light enough so that it corresponds to the virtual tool that is manipulated. The refresh rate of the simulation has to be extremely high (at least $500 \mathrm{~Hz}, 1 \mathrm{kHz}$ if better) in order to fulfill the high temporal accuracy requirements of our tactile sensors. The haptic device must be able to exert intensive forces to if one wants to simulate rigid objects. If the force isn't strong enough, then the user will fill that the objects' surface is soft.

\section{- Exoskeletons}

These devices are less disseminated than the previous class, but they offer a much higher level of realism by giving feedback to entire subpart of the human body like hands. CyberGrasp, Utah/MIT Dextrous Hand Master, and the Rutgers Master II are example of this kind of devices. Most of the exoskeletons available nowadays are hand exoskeletons. Indeed, most of the time the user only have contacts with the virtual environment with his hands (and of course his feet). However, these devices only support one feedback per finger, and to experience a believable grasping, these devices should be able to provide one feedback per finger segment, so that complex shaped objects can be rendered accurately. Also, all the problems that a occurred for the Arm-like devise are still valid for these ones: weight, accuracy of the force, render rate. For the weight of the device, the problem is even more complex because as the user moves his hand around, he will feel the inertia that is due to the device so except if this is partly handled by a secondary robotic arm (as with the CyberForce), this will contribute to lower the believability of the simulation.

\section{- Tactile displays}

These devices aren't force feedback but rather tactile feedback devices i.e. they give the sense of touch: shape, texture, temperature. Examples are CyberTouch, Fingertip Stimulator Array and Elastic Force Sensor. For this kind of devices, the most important issue is the resolution of the actuator. Indeed, for 
smooth surfaces, not many pins are necessary for simulating the texture, but for finer surfaces, the number of pins will have to increase with the complexity of the texture to be rendered.

Because of the dynamic aspect of haptics (i.e. without motion the concept of haptics is almost meaningless), the dynamic response of the system will determine its level of believability. For example, one can render realistically textures with a PHANTOM even if this device has only a pen as an interface. However, to gain a higher level of believability, the use of exoskeletons for actually grasping the virtual objects is crucial. Moreover, this kind of interfaces will have to gain more accuracy (by adding extra end-effectors for every finger segment) to be as efficient as they should. Eventually, even if this is a less important aspect of the simulation, adding a tactile actuator to the device help reaching the level of believable simulation.

\subsection{Multisensory issues}

A virtual environment is an interactive system in which the user manipulates and experiences a synthetic environment through multiple sensory channels. In a multimodal system (like the one proposed by Nigay and Coutaz for example [36]) communication channels are numerous: voice, gesture, gaze, visual, auditory, haptic etc. Integrating these modalities (multimodal inputs) improves the sense of presence and realism and enhances human computer interaction. Virtual environments using sight, sound and touch are quite feasible, but effects of sensory interaction are complex and vary from person to person. Nevertheless adding several communication channels leads to system complexity, cost, and of integration/synchronization problems. Sensory Fusion is a relatively new topic, for which we need to study two kind of human computer communication channels.

The believability of the sensory input should be considered with the information contained in the virtual environment. Even if the sensory channel gives enough realism, it is not sufficient to make the world believable if the world does not give believable contents. In other points of view, some world presented in text-written novels in the form of the book can be believable with well composed stories.

\section{ECA, Emotion and Personality Simulation}

Nowadays, a lot of interest from both industry and research exists for Virtual Environments (VEs) and Embodied Conversational Agents (ECAs). A lot of new techniques are being developed to improve the simulation in general, add more visual detail and make the interaction between human and VE/ECA more natural. Believability is a measure to help to determine how well these different tech- niques are working. Believability represents the 'outsider' point of view. It is thus a very powerful evaluation tool, since we can use it to make evaluations of different techniques/methods while the evaluations are independent from the underlying techniques. This allows us to compare different approaches and give a meaningful indication of their quality on the level of believability.

In this section we will focus especially on believability and ECAs. Since ECAs are generally modelled after humans (even cartoon characters), one important aspect of their believability is how well an ECA succeeds in being like a human. We believe that the key to believable ECAs is the definition of their personality and their emotions. Although quite some research has been done to describe the influence of emotion and personality on ECAs, the results until now are not very convincing. We see several reasons for this:

1. Psychological models of emotion/personality are not finalised. The exact structure of our emotions/personality is not certain, as well as the way in which emotions and personality interact with our perception, behaviour and expression.

2. When one wants to simulate emotions/personality computationally, one tends to take the model the most suitable for a computational simulation. However, this model is not necessarily the best representation for emotions/personality.

3. Even if there exists a perfect emotion/personality model, it is very difficult to distinguish the resulting behaviour from the emotion that is behind it. Also, other issues interfere with our impression of how emotional an ECA really is, such as its appearance, the surroundings, its capabilities, and so on.

In this section, we will attempt to give some ideas how believability of ECAs can be increased. We will especially focus on the expressiveness of ECAs.

An important control mechanism for ECAs is a personality/emotion simulator. Personality and emotions have a significant effect on how one perceives, thinks and acts (see Figure 1). In this section, we will give a short overview of the different existing techniques for including emotions into perception and reasoning. After this section, we will give some examples of how personality and emotion can play a role in expression.

\subsection{Emotion, Personality and Perception}

There are different scenarios that describe how an emotion is evoked from the perception of one or more events (see Figure 2 for an overview). The process of inducing an emotional response from perceptive data is called appraisal. One of the oldest theories, the James-Lange the- 


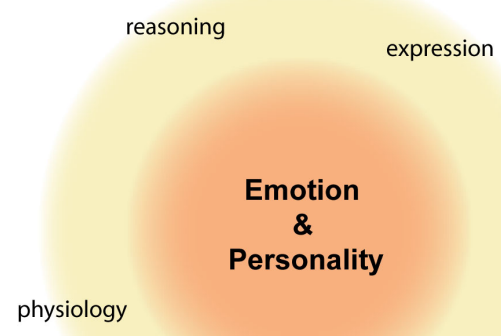

interpretation

perception

\section{Figure 1. Personality and emotion and their} link with ECA system parts.

ory of emotion states that an event causes arousal first and only after our interpretation of the arousal, we experience an emotion. For example:

You are approaching your house and you notice that the door has been forced open. You begin to tremble and your heart starts beating faster. You interpret these physiological changes as being part of fear. You then experience fear.

The Cannon-Bard theory of emotion [7] states that emotion and the physiological response happen at the same time and unrelated from each-other. For example:

You are approaching your house and you notice that the door has been forced open. You begin to tremble and your heart starts beating faster. At the same time you experience fear.

The Schachter-Singer scenario [44] says that an event causes arousal, but that the emotion follows from the identification of a reason for the arousal. For example:

You are approaching your house and you notice that the door has been forced open. You begin to tremble and your heart starts beating faster. You realise that there might be a burglar in your house, which is a dangerous situation. Therefore you experience fear.

The Lazarus theory of cognitive emotion [31] states that both arousal and emotion are invoked separately by a thought following an event. For example:

You are approaching your house and you notice that the door has been forced open. You realise that there might be a burglar in your house, which is a dangerous situation. You begin to tremble and your heart starts beating faster. At the same time you experience fear.

Finally, the Facial Feedback hypothesis [6, 30] says that emotion is the experience of changes in the facial muscle configuration. This result has also been shown by Ekman et al. [16]. For example:

You are approaching your house and you notice that the door has been forced open. Your eyes widen and your mouth corners move backwards. You interpret this facial expression as fear. Therefore you experience fear.

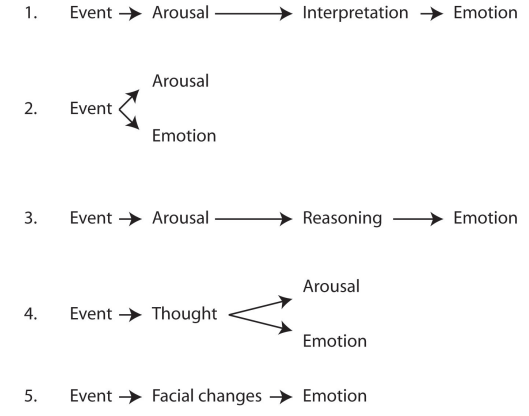

Figure 2. Five scenarios to describe the path from event to emotion: (1) JamesLange (2) Cannon-Bard (3) Schachter-Singer (4) Lazarus (5) Facial Feedback.

In emotion simulation research so far, appraisal is popularly done by a system based on the OCC model [37]. This model specifies how events, agents and objects from the universe are used to elicit an emotional response depending on a set of parameters: the goals, standards and attitudes of the subject. Since the emotional response is generated from a cognitive point of view, this type of appraisal is called cognitive appraisal and it corresponds closely with Lazarus' emotion theory (not taking into account the physiological response).

When one wants to develop a computational model of appraisal, not all of the above mentioned scenarios are suitable to take as a basis, especially those scenarios where arousal plays a crucial role in the determination of the emotional response (ECAs do not yet have a physiology). This rises the question if it is possible to develop a computational model of appraisal that has a high believability.

On the level of personality, one could consider the goals, standards and attitudes of the OCC model as a domaindependent 'personality'. However, personality can also be modelled in a more abstract, domain-independent way [18, 
10]. Egges et al. [13] discusses how a link between multidimensional personality models and the OCC appraisal model can be established.

\subsection{Emotion, Personality and Reasoning}

The effect of personality and emotion on agent behaviour has been researched quite a lot [40], whether it concerns a general influence on behaviour [34], or a more traditional planning-based method [24]. Also, rule-based models [2], probabilistic models [3, 8] and fuzzy logic systems [17] have been developed. In the case of real human beings there are still many questions regarding how emotion influences our behaviour, but in the field of Neuroscience, work has been done that partly describes the relationship between emotions and the brain $[32,11]$.

\subsection{Emotion, Personality and Expression}

The expression of emotions has been widely researched, and the most wellknown research is the work done by Ekman $[14,15]$. Not only will personality and emotion have an effect on expressions by the face or body; also physiological changes can be measured according to different emotions. Furthermore, emotions and personality have an important effect on speech $[45,46]$. In the following two sections, we will concentrate on the relationship between emotions, personality and face/body animation. Also, we will give some examples on how to improve the believability of an ECA using emotions and personality.

\subsection{Believable Facial Animation}

When communicating with an ECA, the dialogue itself is only a small part of the interaction that is actually going on. In order to simulate human behaviour, all the non-verbal elements of interaction should be taken into account. An ECA can be defined by the following parts:

- Appearance (face model, age, race, etc.)

- Behaviour (choice of non-verbal behaviour, accompanying speech)

- Expressiveness of movement (amplitude, tempo, etc.)

Other information can also be important like the cultural background or the context.

Facial animation synchronised with speech can be improved by different factors such as non-verbal actions, speech intonation, facial expression consistent with the speech and the context, and also facial expressions between speech sequences. All this information helps to increase the believability of facial animation for ECAs. The main problem is to determine when and how this kind of non-verbal behaviour should be expressed. Finally, one of the most important points for increasing believability of facial and body animation is the synchronisation between verbal and non-verbal expressions $[39,48]$.

The following types of non-verbal behaviours have a notable influence on the believability of ECAs:

- Gaze: Eyes and head movement play an important role in non-verbal communication. There are rules that describe how eye and head movements are related to the action that is performed. A lot of study has be done in this field [22] that proves the importance of gaze in the communication process. Smid and al. [48] has studied a recorded sequences of real speaker for building a statistical model. This study reveals the importance of head motions during speech.

- Eyebrows: Eyebrow movements are be very important because specific movements during speech are made to stress parts of the contents. Also, eyebrow movements are used in emotions and other expressions, such as uncertainty.

- Expression dynamics: Finally, facial expression timing and dynamics contain a lot of information. Facial expression dynamics change depending on the emotion or personality.

In order to synthesize facial motions, we use a facial animation technique based on the MPEG-4 standard. The details of the facial deformation algorithm are explained in [27]. For defining the visemes and expressions, we use the technique described by Kshirsagar et al. [28]. Here, a statistical analysis of the facial motion data reflects independent facial movements observed during fluent speech. The resulting high level parameters are used for defining the facial expressions and visemes. This facilitates realistic speech animation, especially blended with various facial expressions. In order to generate believable facial animation, the following steps are taken:

- Generation of speech animation from text: a text-tospeech (TTS) software provides phonemes with temporal information. Then co-articulation rules are applied [9].

- Expression blending: proper expressions are selected according to the content of the speech. Each expression is associated with an intensity value. An attacksustaindecay-release type of envelope is applied for the expressions and it is blended with the previously calculated co-articulated phoneme trajectories. This blending is based on observed facial dynamics, incorporating the constraints on facial movements wherever nec- 
essary in order to avoid excessive/unrealistic deformations (see Figure 3).

- Periodic facial movements and gaze: Periodic eye-blinks and minor head movements are applied to the face for increased believability.

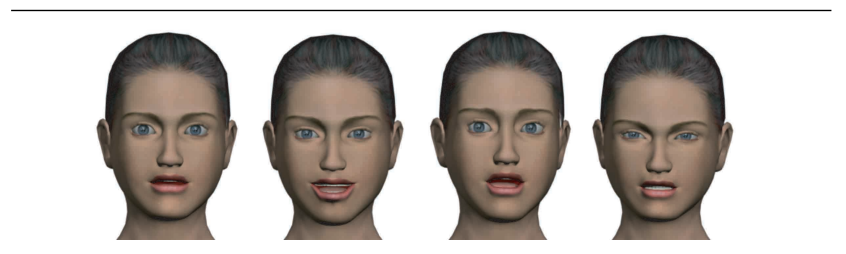

Figure 3. Some examples of facial expressions mixed with speech.

\section{Conclusion}

We have presented in this paper a discussion related to believabilty and virtual environment and specially in terme of Embodied Conversational Agents. A clear description are presented in section 1, presented the different elements of believable environment like immersion, presentation and interaction. We have also presented interfaces related to believability and specally visual sensory feedback, audio and haptic devices.

Personality and emotions should be part of any ECA simulation system. Psychological models of the human mind can help us to determine how we should proceed to include personality and emotions in our ECAs. This aspect are developed in section 3 .

The final implementation of believable virtual world may or may not resemble the human personality/emotion system, but from the point of view of believability this does not matter since the evaluation is independent of underlying technology. In that sense, believability can be seen as the psychology for ECAs.

\section{Acknowledgements}

The research presented has been done in the framework of the European Project HUMAINE (IST-2002-507422).

\section{References}

[1] J. Allen and D. Berkeley. Image method for efficiently simulating small-room acoustics. Journal of Acoustics Society of America, 65:943-950, 1979.
[2] E. André, M. Klesen, P. Gebhard, S. Allen, and T. Rist. Integrating models of personality and emotions into lifelike characters. In Proceedings International Workshop on Affect in Interactions. Towards a New Generation of Interfaces, 1999.

[3] G. Ball and J. Breese. Emotion and personality in a conversational character. In Proceedings of the Workshop on Embodied Conversational Characters, pages 83-84 and 119-121, October 1998.

[4] D. Begault. 3D sound for virtual reality and multimedia. Academic Press Professional, 1994.

[5] J. Borish. Extension of the image model to arbitrary polyhedra. Journal of Acoustics of America, 75:1827-1836, 1984.

[6] R. Buck. Noverbal behavior and the theory of emotion: The facial feedback hypothesis. Journal of Personality and Social Psychology, 38:811-824, 1980.

[7] W. B. Cannon. The james-lange theory of emotion: A critical examination and an alternative theory. American Journal of Psychology, 39:10-124, 1927.

[8] L. Chittaro and M. Serra. Behavioural programming of autonomous characters based on probabilistic automata and personality. Computer Animation and Virtual Worlds, 15(34):319-326, 2004.

[9] M. M. Cohen and D. Massaro. Modelling co-articulation in synthetic visual speech. Springer-Verlag, 1993.

[10] P. T. Costa and R. R. McCrae. Normal personality assessment in clinical practice: The NEO personality inventory. Psychological Assessment, (4):5-13, 1992.

[11] R. J. Davidson, P. Ekman, C. D. Saron, J. A. Senulis, and W. V. Friesen. Approach-withdrawal and cerebral asymmetry: emotional expression and brain physiology. Journal of Personality and Social Psychology, 58:330-341, 1990.

[12] P. Doyle. Believability through context using "knowledge in the world" to create intelligent characters. In Proceedings of the first international joint conference on Autonomous agents and multiagent systems, pages 342-349. ACM Press, 2002.

[13] A. Egges, S. Kshirsagar, and N. Magnenat-Thalmann. Generic personality and emotion simulation for conversational agents. Computer Animation and Virtual Worlds, 15(1):1-13, 2004.

[14] P. Ekman. Emotion in the human face. Cambridge University Press, New York, 1982.

[15] P. Ekman. Approaches to emotion, chapter Expression and the nature of emotion, pages 319-343. Lawrence Erlbaum, Hillsdale, N. J., 1984.

[16] P. Ekman, R. W. Levenson, and W. V. Friesen. Autonomic nervous system activity distinguishes among emotions. Science, 221:1208-1210, 1983.

[17] M. El-Nasr, T. Ioerger, and J. Yen. A pet with evolving emotional intelligence. In Proceedings of Autonomous Agents99, 1999.

[18] H. J. Eysenck. Biological dimensions of personality. In L. A. Pervin, editor, Handbook of personality: Theory and research, pages 244-276. New York: Guilford, 1990.

[19] T. Funkhouser, J. Jot, and N. Tsingos. Sounds good to me. In computational sound for graphics, virtual reality and interactive systems, SIGGRAPH 2002 Conference Proceedings, 2002. 
[20] T. Funkhouser, P. Min, and I. Carlbom. Real-time acoustics modeling for distributed virtual environments. In $S I G$ GRAPH 1999 Conference Proceedings, 1999.

[21] T. Funkhouser, N. Tsingos, I. Carlbom, G. Elko, M. Sondhi, J. West, G. Pingali, P. Min, and A. Ngan. A beam tracing method for interactive architectural acoustics. Journal of the Acoustical Society of America, 2004.

[22] M. Garau, M. Slater, V. Vinayagamoorthy, A. Brogni, A. Steed, and M. A. Sasse. The impact of avatar realism and eye gaze control on the perceived quality of communication in a shared immersive virtual environment. In SIGCHI, 2003.

[23] J. Huopaniemi. Virtual acoustics and 3d sound in multimedia signal processing, 1999. Thesis.

[24] M. Johns and B. G. Silverman. How emotions and personality effect the utility of alternative decisions: a terrorist target selection case study. In Tenth Conference On Computer Generated Forces and Behavioral Representation, May 2001.

[25] M. Kleiner, B.-I. Dalenbck, and P. Svensson. Auralization - an overview. Journal of the Audio Engineering Society, 41:861-875, 1993.

[26] A. Krokstad, S. Strom, and S. Sorsdal. Calculating the acoustical room response by the use of a ray tracing technique. Journal of Sound Vibration, 8:118-125, 1968.

[27] S. Kshirsagar, S. Garchery, and N. Magnenat-Thalmann. Deformable Avatars, chapter Feature Point Based Mesh Deformation Applied to MPEG-4 Facial Animation, pages 33-43. Kluwer Academic Publishers, 2001.

[28] S. Kshirsagar, T. Molet, and N. Magnenat-Thalmann. Principal components of expressive speech animation. In Computer Graphics International, pages 59-69, 2001.

[29] A. Kulowski. Algorithmic representation of the ray tracing technique. Applied. Acoustics, 18:449-469, 1984.

[30] J. Lanzetta, J. Cartwright-Smith, and R. Kleck. Effects of nonverbal dissimulation on emotion experience and autonomic arousal. Journal of Personality and Social Psychology, 33:354-370, 1976

[31] R. S. Lazarus. Emotion and Adaptation. Oxford University Press, New York, 1991.

[32] J. E. LeDoux and W. Hirst. Mind and Brain: Dialogues in Cognitive Neuroscience. Cambridge University Press, 1986.

[33] J. C. Lester and B. A. Stone. Increasing believability in animated pedagogical agents. In Proceedings of the first international conference on Autonomous agents, pages 16-21. ACM Press, 1997.

[34] S. Marsella and J. Gratch. A step towards irrationality: Using emotion to change belief. In Proceedings of the 1st International Joint Conference on Autonomous Agents and MultiAgent Systems, Bologna, Italy, July 2002.

[35] C. Martinho and A. Paiva. Pathematic agents: rapid development of believable emotional agents in intelligent virtual environments. In Proceedings of the third annual conference on Autonomous Agents, pages 1-8. ACM Press, 1999.

[36] L. Nigay and J. Coutaz. A design space for multimodal systems - concurrent processing and data fucion. In INTERCHI'93, pages 172-178, 1993.
[37] A. Ortony, G. L. Clore, and A. Collins. The Cognitive Structure of Emotions. Cambridge University Press, 1988.

[38] R. Pausch, D. Proffitt, and G. Williams. Quantifying immersion in virtual reality. In Proceedings of the 24th annual conference on Computer graphics and interactive techniques, pages 13-18. ACM Press/Addison-Wesley Publishing Co., 1997.

[39] C. Pelachaud and M. Bilvi. Computational Model of Believable Conversational Agents, chapter Communication in MAS: background, current trends and futur. Marc-Philippe Huget, Springer-Verlag, 2003.

[40] P. Piwek. An annotated bibliography of affective natural language generation. Technical report, University of Brighton, July 2002.

[41] G. Robertson, M. Czerwinski, and M. van Dantzich. Immersion in desktop virtual reality. In Proceedings of the 10th annual ACM symposium on User interface software and technology, pages 11-19. ACM Press, 1997.

[42] L. Savioja, J. Huopaniemi, L. Lokki, and R. Vnnen. Virtual environment simulation - advances in the diva project. In ICAD'96, 1996.

[43] L. Savioja, J. Huopaniemi, T. Lokki, and R. Vnnen. Creating interactive virtual acoustic environments. Journal of the Audio Engineering Society, 47(9):675-705, 1999.

[44] S. Schachter and J. Singer. Cognitive, social and physiological determinants of emotional state. Psychol. Rev., 69:379399, 1962.

[45] K. R. Scherer. Personality inference from voice quality: The loud voice of extroversion. European Journal of Social Psychology, 8:467-487, 1978.

[46] K. R. Scherer. Music, language, speech, and brain, chapter Emotion expression in speech and music, pages 146-156. MacMillan, London, 1991.

[47] T. B. Sheridan. Interaction, imagination and immersion some research needs. In Proceedings of the ACM symposium on Virtual reality software and technology, pages 1-7. ACM Press, 2000.

[48] C. Smid, I. Pandzic, and V. Radman. Autonomous speaker agent. In CASA 2004, 17th International Conference on computer Animation and Agent, pages 259-266, 2004.

[49] B. Stevens, J. Jerrams-Smith, D. Heathcote, and D. Callear. Putting the virtual into reality: assessing object-presence with projection-augmented models. Presence: Teleoper. Virtual Environ., 11(1):79-92, 2002.

[50] T. Takala and J. Hahn. Sound rendering. In SIGGRAPH 1992, volume 26 of 2, pages 211-220, 1992.

[51] T. Takala, R. Hnninen, V. Vlimki, L. Savioja, J. Huopaniemi, T. Huotilainen, and M. Karjalainen. An integrated system for virtual audio reality. In 100th Convention of the Audio Engineering Society, 1996. preprint 4229.

[52] D. Zeltzer. Autonomy, interaction, and presence. Presence: Teleoper. Virtual Environ., 1(1):127-132, 1992. 